\title{
Investigation and analysis of deterioration in high pressure compressors due to operation
}

\author{
J. Marx $\cdot$ J. Städing $\cdot$ G. Reitz $\cdot$ J. Friedrichs
}

Received: 15 November 2013/Revised: 14 May 2014/ Accepted: 25 June 2014/Published online: 30 July 2014

(C) The Author(s) 2014. This article is published with open access at Springerlink.com

\begin{abstract}
Due to environmental and operational effects, the deterioration of all gas path-related jet engine components is a highly influencing parameter leading to an increase in exhaust gas temperature and specific fuel consumption over time. As a particularly strained engine module, the high-pressure compressor (HPC) is prone to a variety of abrasive and deforming effects that are responsible for a considerable part of overall engine performance losses. During HPC overhaul, new airfoils are typically mixed with reused parts whose refurbishment workscopes typically range from "passed inspection" up to "full leading and/or trailing edge restoration" as well as "tip weld repair". Hence, a wide spread of airfoil geometries with a distinct statistical distribution can be found within an engine after operation. To allow a statistical analysis, a geometric survey on two full ex-service HPC blade sets and an assortment of equivalent new production parts was conducted. The blades were digitalized by a structuredlight 3D scanner in conjunction with a photogrammetry
\end{abstract}

This paper is based on a presentation at the German Aerospace Congress, September 10-12, 2013, Stuttgart, Germany.

J. Marx $(\bowtie) \cdot$ J. Städing

MTU Maintenance Hannover GmbH, Performance Engineering,

Münchner Str. 31, 30855 Langenhagen, Germany

e-mail: jonas.marx@mtu.de

G. Reitz $\cdot$ J. Friedrichs

TU Braunschweig, Institute of Jet Propulsion and

Turbomachinery, Hermann-Blenk-Str. 37, 38106 Braunschweig,

Germany system. Based on the measured three-dimensional data, a CAD model was generated for each blade. Afterwards, airfoil sections on different blade heights were analyzed to generate aerodynamic design parameters such as leading and trailing edge geometries (radius and thickness), their maximum profile thickness, their chord length and their stagger angle. By assessing the statistical results of both used and new parts, the effects of manufacture variations and in-service wear on HPC blade geometry can be compared in detail.
Abbreviations
3D Three-dimensional
CAD Computer-aided design
CSLSV Cycle since last shop visit
EGT Exhaust gas temperature
Eng Engine
HPC High-pressure compressor
LEP Leading edge point
OEM Original equipment manufacturer
OGV Outlet guide vane
PS Pressure side
sfc Specific fuel consumption
SS Suction side
Stg. Stage
TEP Trailing edge point

\section{Symbols}

$h^{*} \quad$ OEM-defined Blade Height

$h / H \quad$ Relative channel height

$l \quad$ Chord length

$r \quad$ Radius

$t \quad$ Thickness

$\lambda$ Stagger angle 


\section{Indices}

LE Leading edge

max Maximum

TE Trailing edge

\section{Introduction}

Over an aircraft engine's time in service its components are exposed to a large number of deterioration effects that inherently oppose design intent. This is especially true for the high pressure compressor where ingested sand, fouling, and operational strains may lead to a variety of geometric changes of the HPC airfoils that stack with existing manufacture deviations. These effects of wear and tear include erosion on pressure and suction surfaces, chord length reduction, deformation of leading and trailing edges, a variation of stagger angle as well as a widening of tip clearances. Their characteristics do typically correlate linearly or regressively with operation time and affect compressor efficiency as well as component matching detrimentally. Consequently, the exhaust gas temperature and specific fuel consumption rise with progressing deterioration, as the engine control compensates deficits in compressor performance via an increased fuel flow to meet thrust requirements.

While the condition of an airfoil in service is always based on the superposition of the aforementioned aspects of deterioration, the usual approach in analytic studies is to concentrate on one or two geometric deviations and their impact on compressor performance. In this regard, the influence of variable chord lengths was investigated by Roberts [10]. For that he arranged blades with full nominal chord next to parts with recontoured leading edges in several alternating assemblies of a transonic compressor rotor. It should be noted that a comparison between the performance of the blading with $50 \%$ new and $50 \%$ modified blades and a completely new blading is not been given. While analyzing the modified blading, he detected no significant difference in performance characteristics between the considered configurations except for near-stall operation. Because of this Roberts implies that the impact of variable chord length on the aerodynamic parameters is not crucial if the blades have been recontoured properly and their chord is above stall chord limit.

Giebmanns et al. [2] analyzed the influence of leading edge and chord length variations on aerodynamic performance of transonic fan blades. Here, the leading edge shape was found to be the dominant factor regarding pressure rise as well as efficiency, whereas recontoured blades with reduced chords lengths still offered decent performance. This effect was mostly attributed to loss production of the upper span shock system and its sensitivity to blunted LEs.
Airfoil manufacture variations were addressed by Goodhand [3] who considered the effect of new part leading edge geometry on incidence range. Although Goodhand observed a considerable manufacture scatter in LE thickness of $10 \%$, he determined no noteworthy effect on mean incidence as a result of these deviations. In this regard, he identified a particularly high sensitivity of sharper leading edges towards flow variation which lead to deleterious effects on operation range. As a consequence, the author offers guidelines for avoidance of this deleterious behavior and introduces an optimization approach for supercritical leading edges. Goodhand furthermore points out that based on his data the scatter in leading edge geometry of new production parts very well exceeds deterioration due to service.

As far as the refurbishment of airfoil geometries of conventional single blade HPC configurations is concerned, there are several restoration procedures that aim for a recovery of performance and stability losses. Guided by engine manual limits and the individual condition of the part, common workscopes for the HPC require a welding of the blade tips, vibro polishing of the surfaces and, if available, recontouring of the leading and trailing edges. These repairs are addressed by well established inspection criteria like blade height, surface roughness, chord length, and LE thickness. However, there are geometry parameters that either cannot be restored (e.g. profile thickness) or that are not even addressed by the engine manual (e.g. stagger angle or LE contour). If found in accordance with the EM limits the blades that show the latter kind of deformation are incorporated in the HPC blade set. Hence, the HPC blading features worn, repaired and new parts after reassembly. Depending on the quality of the airfoil repairs, the stringency of the engine manual limits and the aerodynamic impact of disregarded geometry parameters, it is hardly surprising that the performance of an overhauled compressor may differ considerably from that of a newly manufactured.

The influence of blade deterioration on flow and performance of a representative HP compressor stage was investigated by Krone et al. [5] in a 3D steady-state numerical approach. Krone confirms the influence of operational airfoil degradation on compressor efficiency and pressure gain by showing quantitative results for exservice blade conditions. Performance characteristics were predicted to show an offset towards higher flow coefficients as compared to the new production throttle curves and in addition a change in their slope. This behavior was attributed to a reduced twist of these blades at tip and an overall thinner profile. However, since only samples of investigated used blades are analyzed, Krone raised question whether his approach is statistically sufficient to provide a representative round-up of blade wear and overhauled airfoil conditions. 
A method of geometry acquisition which allows for a robust statistical analysis of airfoil deviations is introduced by Lange et al. [6] using a parametric blade model. The described procedure is based on the commonly utilized structured light 3D-scanning backed by a photogrammetry system and originally aimed for an evaluation of manufacturing scatter. A sample of 150 new production HPC rotor blades were digitized (i.e. converted into 3D data), parameterized by a vector of 14 aerodynamic profile parameters and compared to the design intent. Ensuing investigations draw on the resulting statistical database for a variety of probabilistic studies concerning the overall performance scatter of newly manufactured compressors $[7,9]$ and the impact and interactions of non-axisymmetric blading [8].

To gain improved knowledge of the statistical occurrences of airfoil deterioration symptoms on ex-service blades, a geometric examination of two complete HPC blade sets was carried out which follows an approach similar to that of Lange et al. [6]. While the present work also relies on a parametric blade model to parameterize 3D digitized airfoil geometries, it concentrates more on a clear separation of manufacture scatter and degradation effects. Thus, a database for rated HPC blade deterioration is created that provides ongoing numerical research with highly needed input and focus. Please note, that the foremost objective of this study is not to identify root causes but to evaluate the appearance and characteristics of geometry deviations.

\section{Experimental setup and metrology}

For this investigation more than 1,400 used compressor airfoils were digitized by means of structured-light $3 \mathrm{D}$ scanning with an accurancy of $8-10 \mu \mathrm{m}$ in conjunction with a photogrammetry system and translated into finely resolved point clouds. The blades in question were derived from two full sets of ten-stage HPC assemblies that originate from intermediate thrust high bypass turbofans. Both engines were brought into service in middle and southern Europe as well as the Mediterranean area by the same operator on known short-to-medium-range air routes. As far as the comparability of these two engines is concerned, only the on-wing time differs noteworthy (engine I: approx. 5000 CSLSV; engine II nearly 3200 CSLSV). The composition of each individual blade set is arbitrary to a certain extent which is common for engines that already received at least one shop visit. This means that with the exception of the operation time indicated by the CSLSV the background of each airfoil in terms of previous inspection results and individual report is not fully known. Whether a particular blade received a specific repair in its past or was deemed serviceable without any need for refurbishment during previous shop visits is not documented. In addition to ex-service blade sets, 300 new production airfoils (30 parts per stage) were scanned to consider manufacture variations for each inspection characteristic.

To avoid misalignments of scanned blades as observed by Holtzhausen [4], all data sets were aligned to their dovetail pressure faces and an additional plane of symmetry situated in the root area. For this paper the full dovetail geometry had to be scanned and analyzed as well. This orientation allows for a subsequent numerical simulation of in-flight airfoil positions due to centrifugal forces.

The parameterization of the blade geometry is carried out by means of a parametric compressor blade model which has been developed at the Institute of Jet Propulsion and Turbomachinery (TU Braunschweig). Given an identical alignment of the root, all airfoils will be segmented in 19 equally spaced conic sections from 5 to $95 \%$ blade height and 3 stage-specific sections which refer to the OEM inspection in the engine manual (see Fig. 1). For each of these sections the leading and trailing edge geometries (thickness and radius), the maximum profile thickness as well as the stagger angle are determined.

Starting with the definition of the camber lines, the parameterization algorithm uses a rolling ball method on each profile section, fitting tangential circles through every measuring point and into the corresponding profile contour. Consequently, the number of circles depends on the resolution of the point cloud which typically ranges from 1,4 to $1,7 * 10^{3}$ points per profile section (see Fig. 2). To ensure that deteriorated edge geometries do not influence the algorithm, there is an abort criterion by fitting the circles through the profile contour. This criterion terminates the generation of the circles near the leading and trailing edge. In a second step, the circle centers are connected via a 6th degree polynomial fit that already generates the largest portion of the camber line between the centers of the

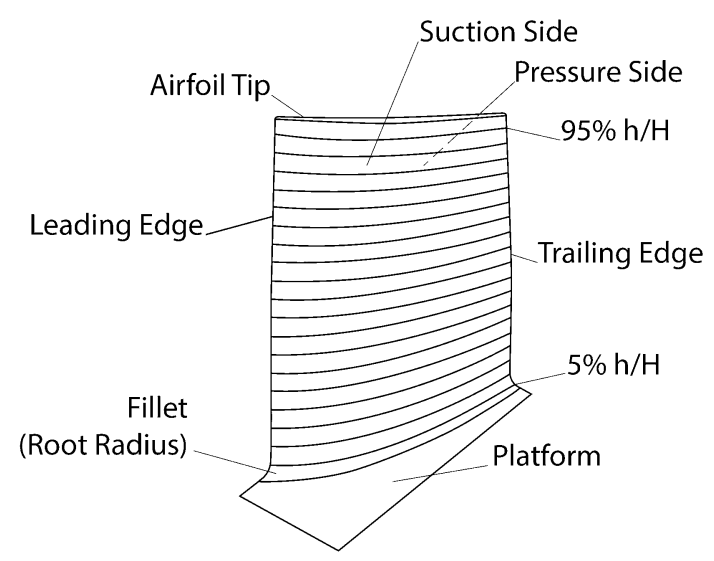

Fig. 1 Airfoil nomenclature 


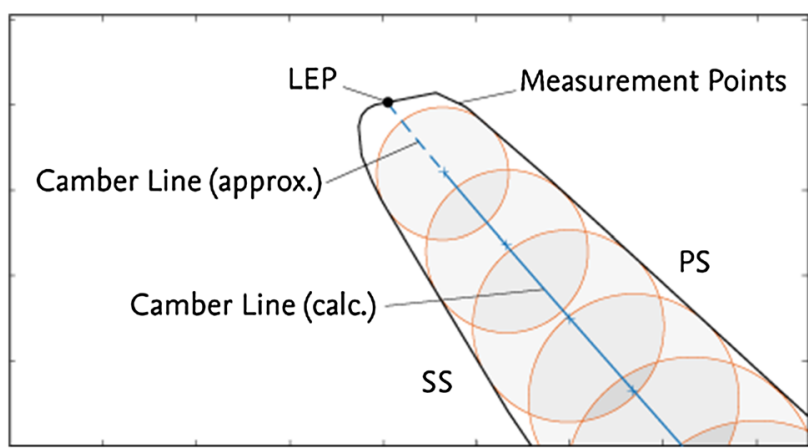

Fig. 2 Creation of the camber line

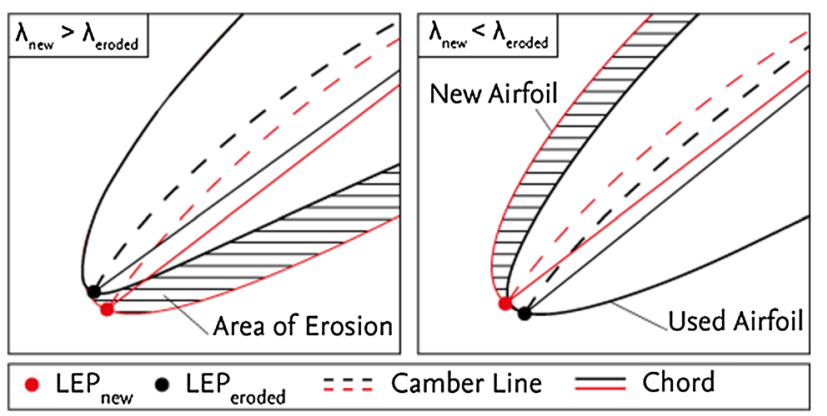

Fig. 3 Asymmetric eroded leading edge

leading and the trailing edge circles. To close the remaining distance between camber line and profile contour, the line is extrapolated upstream and downstream, continuing the curvature of the adjacent $1 \%$ at LE and TE. The resulting intersections between extended camber line and profile contour present the leading edge (LEP) and trailing edge points (TEP), respectively. Per definition, the chord length of a given profile section is the shortest distance between its LEP and TEP. The stagger angle is situated between said chord and the machine axis.

The influence of an asymmetric eroded leading edge on the stagger angle can be seen in Fig. 3. In case of stronger removal of material at the pressure side the leading edge point will shift towards the suction side consequentially decreasing the stagger angle. In contrast, the calculated stagger will be increased if suction sided-erosion is predominant. Since the chord length will also be reduced the more pronounced the single-sided erosion is, the change of stagger angle and chord reduction are coupled by this effect.

Aside from chord-related parameters, the construction of the camber line also provides values of profile thickness as well as the LE and TE radii. Maximum thickness of a profile corresponds with the diameter of the largest circle that has been created for camber line definition. The leading edge and trailing edge radii are analogously defined by the foremost and the rear circle, respectively. The thickness of the leading and trailing edges is determined at 5 and $95 \%$ chord length orthogonally to local camber line slope.

Please note, that all measured profile parameters presented in this paper have been normalized with the corresponding reference geometry, which is the new part mean value of the described parameter at the investigated blade span.

\section{Description of geometric variations}

Visualizations of HPC blade deterioration in this paper are provided in terms of single stage and overall compressor characteristics. While both display formats feature a single profile parameter which is plotted over channel height $(h / H)$, they are otherwise very distinct to fulfill specific requirements. The single stage plots not only provide detailed information on the general degradation behavior of a given parameter in a specific stage, they also address the geometric variations of used and new airfoils. Aiming at a clear separation of manufacture scatter and degradation effects, the single stage plots also include standard and maximum deviation of deteriorated blades as well as new production parts. By contrast, overall compressor charts are narrowed down to mere average data, but allow to follow a possible propagation of deterioration mechanisms from front stage to rear stage.

\subsection{Behavior of single stage deterioration}

Considering the degradation of compressor performance and the consequential increase of fuel flow and exhaust gas temperature, it only seems natural that the actual condition of blades in a matured HP jet engine compressor is essentially affected by operation. Ebmeyer et al. [1] describes that this is primarily due to erosion which is caused either naturally (e.g. sand ingestion) or by anthropogenic effects (e.g. industrial emission). The scale and appearance of deterioration by all means depends on the engine's time on-wing, flight ratio and area of operation in particular. For the individual airfoil it is affected by material properties, particle size as well as flow conditions (e.g. incidence, velocity etc.). Deterioration may be further increased by fouling and additional operational strains such as tip rubbing or the untwisting of blades due to aerodynamic loads.

However, it has to be taken into account that the compressor blading is just as well characterized by manufacture deviations and repair-related geometrical variations. While the latter influence cannot be a subject of this investigation due to a lack of documentation on airfoil repairs for the selected engines, a comparison of operational and 
manufacture shape variations is given in the following. Presented are the leading edge thickness, the maximum airfoil thickness and the stagger angle for ex-service and new production airfoils of a front (2nd), an intermediate (6th), and the rear (10th) compressor stage in terms of radial plots which are customary in the field of turbomachinery. Please note, that max. profile thickness and stagger angle usually remain unaffected by standard blade repairs and thus are expected to be solely subject to deterioration and manufacture scatter.

\subsubsection{Front stage (2nd HPC stage)}

Characteristics of leading edge thickness, maximum profile thickness, and stagger angle for used and new airfoils of the 2nd HP compressor stage are presented in Fig. 7a, d and g, respectively. All data have been corrected with the new production mean value of the corresponding cross-section which, is set to $100 \%$ from hub to tip. New production standard and maximum deviation are provided in terms of grey-shaded areas around the mean. Equivalent values of the ex-service blades are portrayed by the solid (used average) and dashed (used maximum/minimum) lines. Refer to the attached error bars for used blade standard deviation. In addition to the radial plots, Figs. 4, 5, and 6 depict actual geometries of sensitive regions of this stage for specific spans in detail. Here, the average new part is portrayed by the red line. The ex-service profiles that were found the most and the least deteriorated are illustrated by the dash-dotted and dashed lines, respectively.

2nd stage HPC leading edge is severely affected by deterioration, showing an average decrease in thickness of up to $15 \%$ ranging from hub to $65 \%$ blade height (see Fig. 7a). Above $75 \%$ span leading edge thickness recedes to new production level, though standard and overall deviations of used parts still exceed manufacture scatter by a decent amount. The average ex-service airfoil appears to be particularly eroded at $55 \%$ span where the profile is reduced to even less than $80 \%$ of its original thickness. Although not shown here, the average LE radius was found to behave analogously to the LE thickness distribution. From hub to $70 \%$ span the LE not only becomes thinner but also considerably sharper, i.e. the LE radius decreases. In this regard, Fig. 4 grants a detailed view on the local situation and gives an idea of how the leading edge at $55 \%$ $h / H$ is thinned out more or less symmetrically by operation. For easier comparison the profiles are shifted to the same LEP in this figure. At this section the average radius is reduced to approximately $77 \%$ of its reference value. In the tip region (above $80 \% \mathrm{~h} / \mathrm{H}$ ), however, opposing observations can be made. Here, the leading edge was found to be blunted with radii of up to $110 \%$ of new production standard.

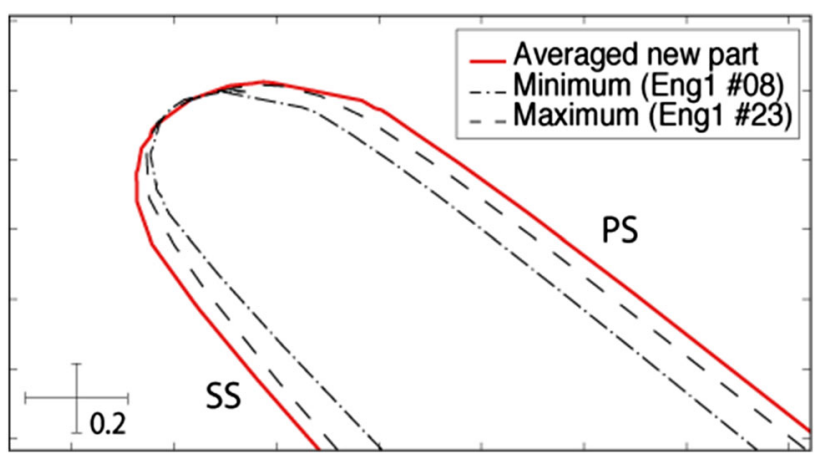

Fig. $4 \mathrm{Min} / \mathrm{max}$ deviation of leading edge from averaged new parts (HPC Stg. 2, $55 \% h / H$ )

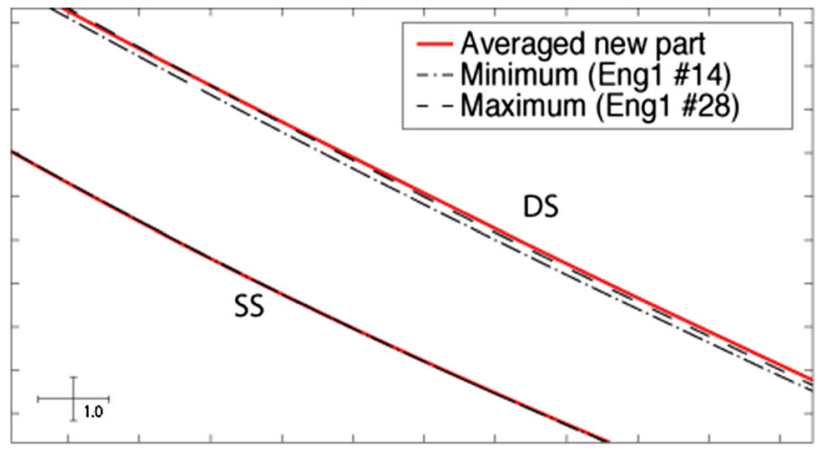

Fig. $5 \mathrm{Min} / \max$ deviation of max. profile thickness from averaged new parts (HPC Stg. 2, $65 \% \mathrm{~h} / \mathrm{H}$ )

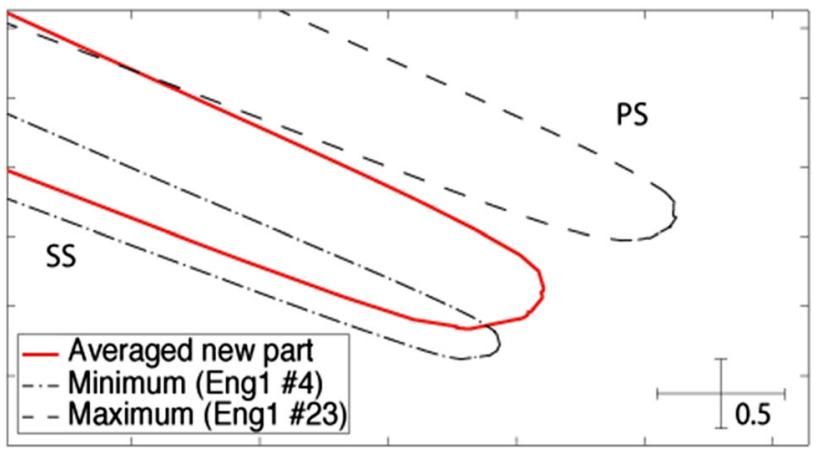

Fig. $6 \mathrm{Min} / \mathrm{max}$ deviation of stagger angle from averaged new parts (HPC Stg. 2, $85 \% h / H$ )

Unlike the aforementioned characteristics the maximum profile thickness, illustrated in Fig. 7d, seems to be less sensitive to erosion as far as absolute variations are concerned. Even so, there are regions from 10 to $30 \%$ span and from 50 to $70 \%$ span where the relative averaged maximum thickness of used airfoils falls below new part standard deviation about $1-2 \%$. The maximum reduction in profile thickness from average used to average new in these regions ranges from 2 to $4 \%$, respectively. A 


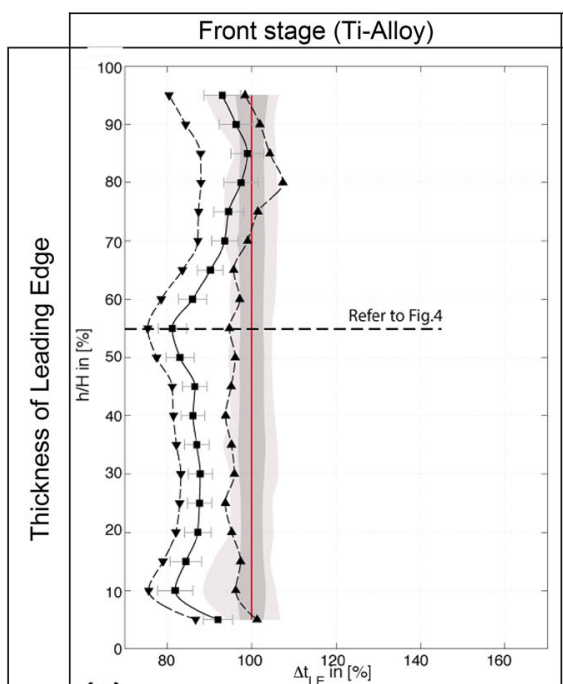

(a)

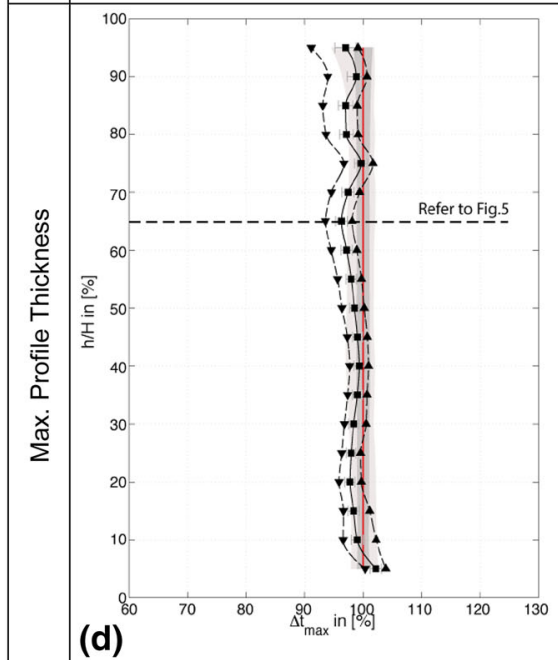

(b)

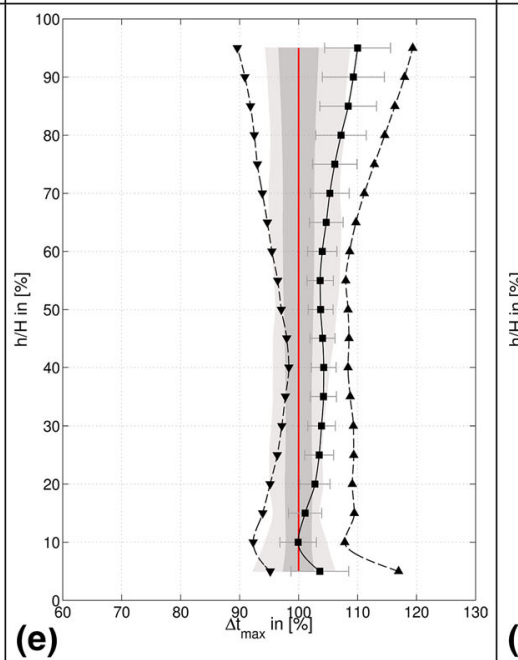

(c)
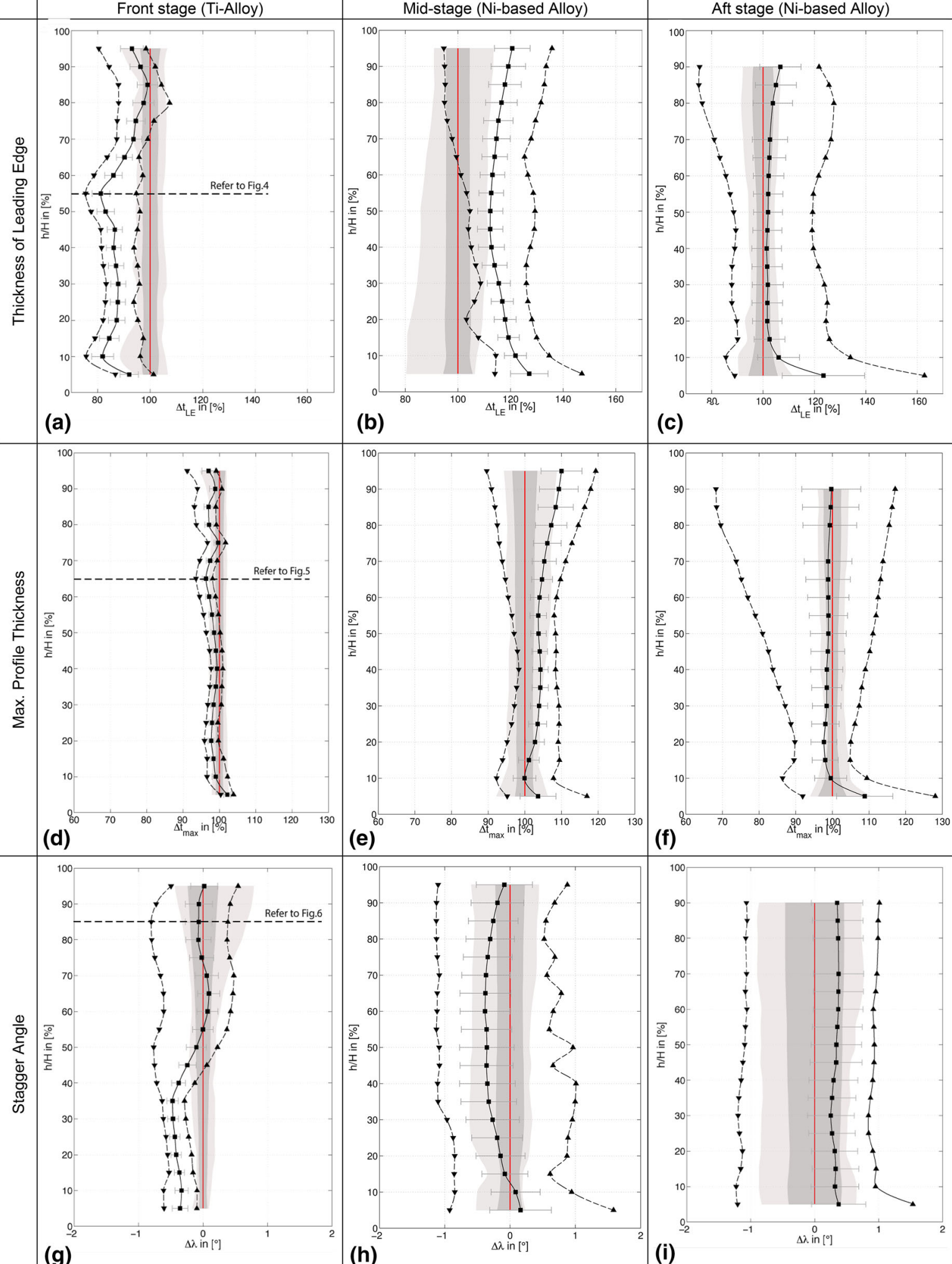

(g)
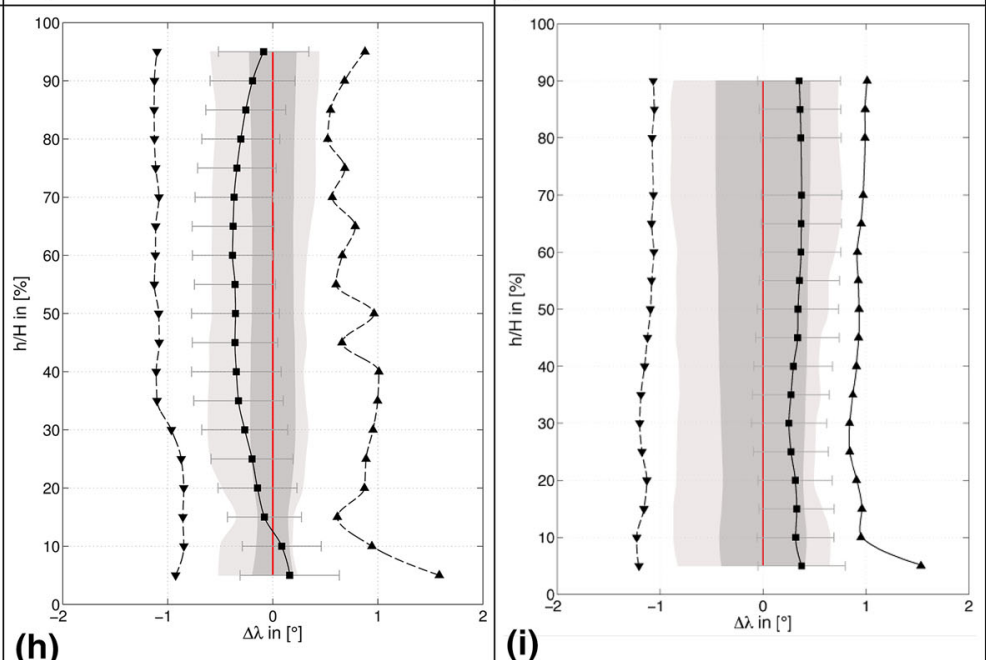

(i)

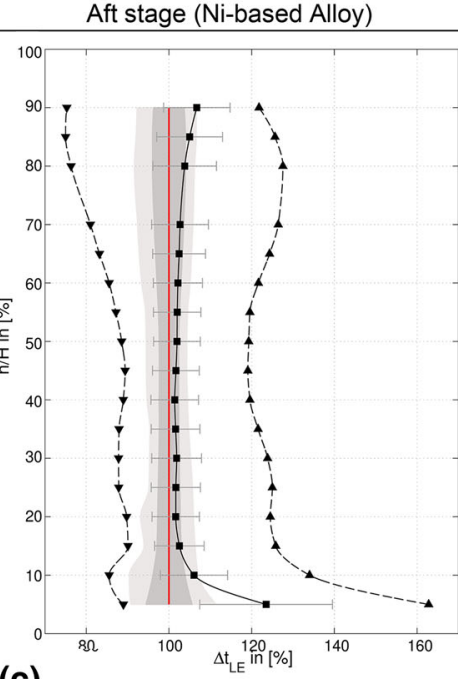

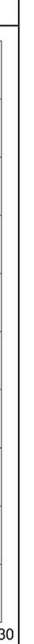

- Mean (Used) _ _ Standard Deviation (Used)

、 Minimum (Used)

- Maximum (Used)

Averaged New Part $\quad$ Standard Deviation (New)

Production Spread (New)

Fig. 7 Single stage deterioration of selected parameters of Engine 1 
likewise behavior can be found in the tip region above $70 \% \mathrm{~h} / \mathrm{H}$, albeit with one striking irregularity at $75 \%$ span. Nevertheless, the maximum reduction of profile thickness is about $9 \%(95 \% h / H)$ and its largest value is at $5 \% \mathrm{~h} / \mathrm{H}$ with $104 \%$. Note that by definition an increase in maximum thickness like that in the near-hub region cannot be caused by natural deterioration. As the LE thickness distribution (see Fig. 7a) shows a comparable trend near the hub, tending towards higher values, all measurement data at $5 \% \mathrm{~h} / \mathrm{H}$ were presumably already influenced by the fillet area. While it can only be speculated given the lack of information on airfoil biography a possible explanation would be a change in production scatter in the time period between production of the examined used and new airfoils. In this sense, due to probable manufacturing variations similar and not identical geometries are compared to each other.

The characteristics of the used blades' stagger angle which again are referenced to the new production average show an unexpected progression (see Fig. 7g). Beginning at the hub to about $35 \%$ span the ex-service stagger angle is uniformly up to $0.5^{\circ}$ lower than reference. Beyond that this deficit recedes and falls within new part standard deviation from mid-span to casing featuring a mild overshoot around $60 \% \mathrm{~h} / \mathrm{H}$. The largest reduction of the stagger angle can be found at $85 \% \mathrm{~h} / \mathrm{H}$ with $0.8^{\circ}$ (see Figs. 6, 7) while the greatest increase is at $95 \% \mathrm{~h} / \mathrm{H}$ with $0.5^{\circ}$. Given the lack of stagger angle shortfall in the upper half of the span, it is highly unlikely that this trend indicates an untwisting of the blade due to operational stresses. In any case standard and maximum deviations in stagger angle will increase significantly from hub to tip. Nevertheless the scatter between maximum and minimum values in the tip region of the used blades is with more than $1^{\circ}$ clearly greater than the scatter of the new blades, even though their standard deviation is comparable and the mean value of the used blades is inside the standard deviation of the new blades. In addition a greater shift from the mean to the minimum values than to the maximum values can be noticed.

\subsubsection{Mid-stage (6th HPC stage)}

Distributions of leading edge thickness, maximum profile thickness, and stagger angle variations of matured intermediate HPC stage blades are presented in Fig. 7b, e and h, respectively. Again, all quantities have been referenced to new production average of the appropriate profile section.

Referring to Fig. 7b, the severity of erosion of the 6th stage leading edges appears to be on par with that of the front compressor stage. However, whereas the degradation findings on the 2nd stage indicated collectively sharpened airfoils, the leading edges of the intermediate stage have been consistently blunted by operation. This is illustrated in Fig. $7 b$ with the progression of the ex-service mean of LE thickness which ranges from 112 to $128 \%$ of reference depending on span. In this regard, some airfoils were even found at $130 \%$ thickness and beyond with values tending to be slightly higher in the endwall regions. In addition, the leading edge radii are similarly affected by operation (compare Fig. 8a) showing considerable and consistent increase over the entirety of the blade height. Note, that these findings do not contradict the theory of abrasive degradation, as the definition of leading edge thickness very well allows for values over $100 \%$ of new production standard (compare Sect. 2).

This is not true for the maximum profile thickness though which is depicted in Fig. 7e. As a matter of fact, the maximum thickness of used 6th airfoils appears to be higher than new part reference, albeit with vastly increased standard and overall deviations towards the tip. Hereby the greatest scatter is localized at $95 \% \mathrm{~h} / \mathrm{H}$ with the 90 and $120 \%$ maximum profile thickness. As noted above, this increase by definition alone cannot be caused by deterioration and is presumably production related. However, these findings certainly emphasize the independence of certain profile parameters as well as the utter diversity matured high pressure compressors display.

That the condition of compressor airfoils of a given stage in service may also vary from engine to engine is substantiated by the 6th stage trailing edges of both analyzed compressors. Whereas TE thickness and radius for Engine 1 are slightly decreased as compared to reference but are otherwise within new production standard deviation, Engine 2 features rather blunted edges by tendency. It is of importance to note that although the average trends for both engines disagree, the maximum range (min. to max.) of both TE radius and thickness fits surprisingly well over the majority of the span. A possible explanation could be the execution of a trailing edge weld repair on a divergent amount of blades for each motor. In this case, the HPC of Engine 2 would be expected to contain the greater number of TE welded blades pushing the overall mean of related profile parameters towards higher values. As long as only a limited portion of the set is treated thusly, the extreme values of said parameters will be considerably less affected than their average.

The radial plot in Fig. $7 \mathrm{~h}$ provides information on the characteristics of the 6th stage stagger angle in relation to new production average. Starting at $25 \%$ span the exservice stagger angle falls below manufacture standard deviation and exhibits a minimum of $-0.4^{\circ}$ at $60 \%$ $h / H$. With diminishing distance to the blade tip this deficit recedes again, reaching design intent at $90 \%$ span. Hereby the smallest stagger angle shows a constantly progression over the blade height of $-1^{\circ}$. The maximum stagger angle 


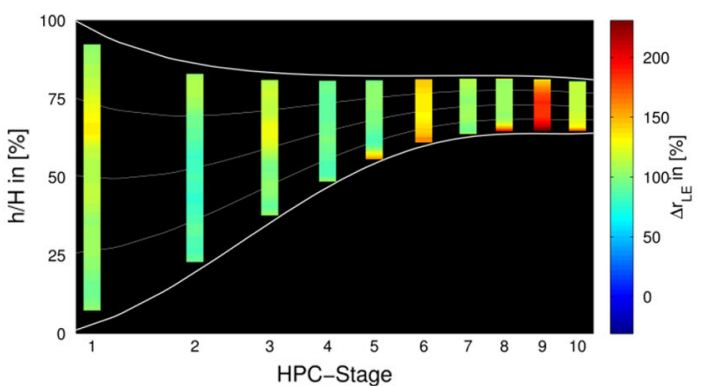

(a) Radius of Leading Edges

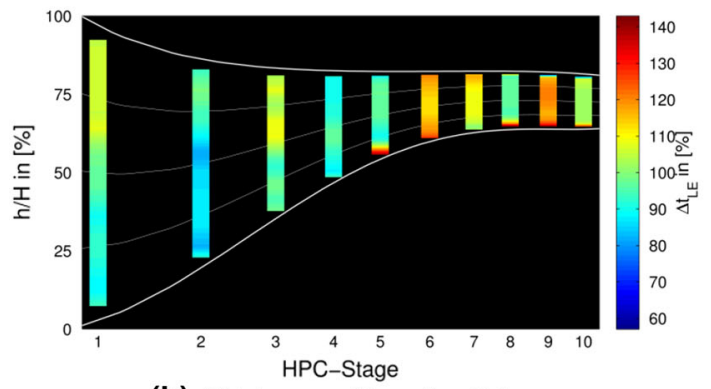

(b) Thickness of Leading Edges

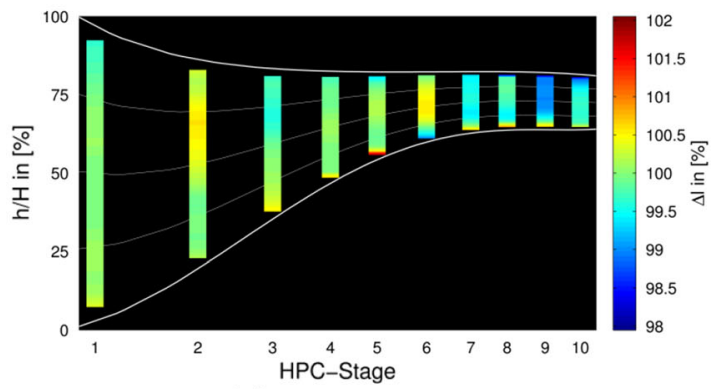

(c) Chord Length

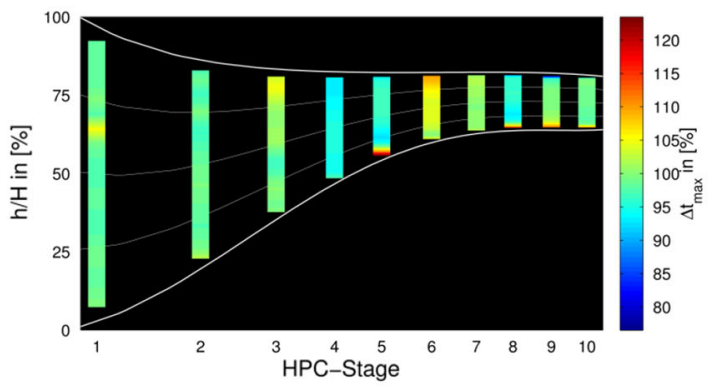

(d) Maximum Profile Thickness

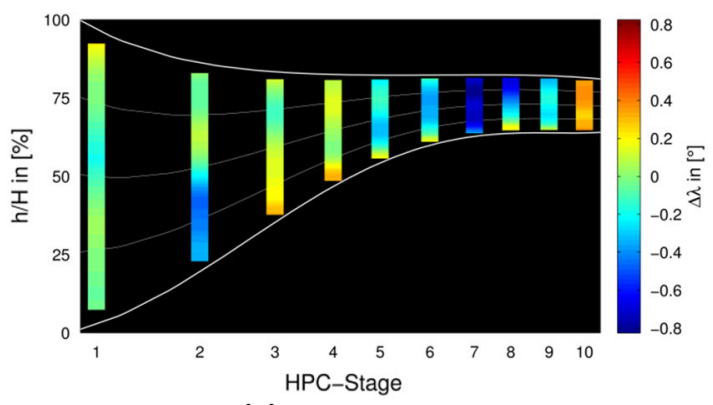

(e) Stagger Angle

Fig. 8 Overall HPC deterioration has no comparable constantly progression and has a maximum of $+1,5^{\circ}$ at $5 \% \mathrm{~h} / \mathrm{H}$. Similar to the 2 nd stage, the shift between minimum and mean values seems to be greater than between mean and maximum value. In analogy with the 2nd stage findings this behavior is deemed atypical for an operational untwisting of the blade given that the increase in stagger angle does not rise steadily with blade span. Again, a possible explanation could be deformations of the airfoil that change the alignment of the leading or trailing edge points and thus the chord pitch. Otherwise variations in manufacturing procedures of the examined ex-service and new production airfoils could influence the stagger angle more or less randomly. Aside from the average trend, ex-service standard, and maximum deviation clearly exceeds corresponding manufacture scatter over the entire blade span.

\subsubsection{Aft stage (10th HPC stage)}

Figure 7c, f and i present spanwise progressions of selected profile parameters for the rearmost HPC stage. Note, that the thinner the airfoils, the larger a scale has to be applied for thickness variations ( $x$-axis) to allow for a complete illustration of the maximum deviations of used parts which increases with ascending stage number. The fact that the magnitude of new production scatter in general appears to be independent from stage number, potentially hints at an overall intensified deterioration of the downstream compressor stages (refer to sect. 3.2).

Like compressor stage 6 the rear stage no. 10 also features airfoils with blunted leading edges from hub to tip. This is confirmed in Fig. 7c showing a slightly but consistently increased LE thickness as compared to new production reference $(+2$ to $+10 \%)$. The degradation is highest in the tip area since the majority of ingested particles that are influenced by the swirl tend to pass at the higher radii. This is especially true this far downstream in the compressor. High values of LE thickness below $10 \%$ span are probably due to the influence of the fillet area (compare with stages 2 and 6). Although not illustrated, the potential bluntness of the 10th stage airfoils is furthermore verified by a considerable increase of ex-service leading edge radii which are at $110-125 \%$ of new production average. The range of standard and maximum deviation of both profile parameters is much greater for used than new parts.

The maximum profile thickness of a generic ex-service 10th stage airfoil as a function of blade height is displayed in Fig. 7f. Also given are the corresponding standard deviation and extreme values (70 and $120 \%$ at $95 \% \mathrm{~h} / \mathrm{H})$ that could be derived from a complete blade set. Much like the previously discussed stages the used 10th stage exhibits 
a deterioration scatter that increases with span and exceeds manufacture variations by far. On average the maximum thickness propagates mostly within new production standard deviation nonetheless. Only in a limited region between 15 and $25 \%$ span abrasive deterioration becomes evident through a somewhat slimmer profile.

The characteristics of the stagger angle of used and new stage 10 airfoils are presented in Fig. 7i. Despite a small and constant shift of the matured airfoils tending towards a higher stagger $\left(+0.3^{\circ}\right)$ which is covered by new part standard deviation, no significant geometric changes due to deterioration are apparent. However, it should be noted that this stage shows a greater shift between minimum and mean value than between mean and maximum value, too. The shift between the minimum and mean value seems to increase with the HPC stages. In addition, the progression of the minimum stagger angle is comparable to stage 6 . It has a constant value over blade height about $-1^{\circ}$. Moreover, the maximum stagger angle has a constant progression, too. The maximum stagger angle increase about $1^{\circ}$.

In summary, the measurements of the 2nd, 6th, and 10th stages HPC rotor show a wide spread of deterioration than initially expected. Although, the thickness of leading and trailing edge as well as the maximum profile thickness show various signs of degradation (either sharper ended or blunted), this erosion is not necessarily concentrated on the tip region (compare Krone et al. [5]). Profile parameters of the 2nd stage like leading edge thickness or stagger angle showing a more significant variation along blade span. While this might be explained in parts by parameter definition it certainly demands attention for the entirety of the span if one would evaluate airfoil deterioration aerodynamically. Standard and overall deviations of ex-service blades, however, do clearly increase from hub to tip. This is true for all investigated profile parameters of all three stages.

\subsection{Behavior of overall HPC deterioration}

To understand the variation of geometry parameters from stage to stage in the analyzed jet engine compressor, the following charts illustrate the overall compressor characteristics of eight parameters in total. Contour plots of LE and TE thickness and radius as well as chord length, stagger angle, and maximum profile thickness for all ten stages of the compressor are presented in Fig. 8a-g. All quantities have been arithmetically averaged over each ex-service blade set and corrected with the new production reference geometry as usual. Contour lines of compressor hub (lower boundary) and casing (upper boundary) as well as for the 25, 50, and $75 \%$ channel height indicate span-wise location.

Profile parameters that define the leading edge geometries for all HPC stages of Engine 1 are given in Fig. 8a and b in terms of LE radius and thickness. While these parameters already have been discussed for stages 2,6 , and 10 in Sect. 3.1, the plots allow for an overview of leading edge deterioration in the overall compressor. At this, front and rear stages appear to react rather differently to operation. Whereas the upstream blade rows (stages 1-5) show slightly sharpened LEs (i.e. values lower than $100 \%$ ), the downstream stages (stages 6-10) feature blunted leading edges to a great extent. Here the deterioration becomes particularly apparent, like in stage 9 .

Where the increase in LE radius and thickness exceeds $30 \%$ of design intent over the entirety of the span. However, a smooth transition of LE deterioration from front to rear cannot be detected. Unexpected in a similar manner is the finding that erosion does not appear to be focused on the blade tip or upper span regions. Only stages 1, 3, 6, and 7 show a gradient span-wise trend in deterioration.

Operational variations in chord length, stagger angle, and maximum profile thickness of used HPC airfoils from new production standard are depicted in Fig. 8c-e for all ten stages. A noteworthy change of chord length (see Fig. 8c) could be identified beginning in stage 7 and continuing further downstream. Here, chord length and overall shortening of the blades ranges from 0.5 to $2 \%$ and correlates well with leading and trailing edge degradation. Hence, it is particularly high in stage 9. An increase in chord length is implausible in terms of deterioration and could be explained by LE/TE weld repair influence. The untwisting of the upper blade region which has been reported by Krone et al. [5] can only be confirmed to some extent for blade rows 7,8 and 9 (compare Fig. 8e). At best it is characterized by a deficit in stagger angle that increases steadily with blade height. As already mentioned in Sect. 3.1 the stagger angle may also be sensitive to leading and trailing edge deterioration since it would relocate LEP and TEP respectively and thus change chord pitch. In addition to possible changes in manufacturing procedure this could explain the irregular distribution of stagger angle variations throughout the compressor. Regions where the maximum profile thickness is considerably impaired by deterioration are sparse and can only be observed in stages 4,5 , and 8 (see Fig. 8d). Interestingly, there is no correlation between leading edge and maximum thickness. The leading edge thickness seems to be dependent on the operation and the repairs whereas the maximum thickness appears to be rather depending on the production. Here, an additional correlation survey will be processed to identify dependent parameters. Note, that a substantial increase of maximum profile thickness as compared to new production reference (like in stage 6) cannot be caused by erosion and thus, has to be traced back to deviations between production charges. 


\section{Conclusion}

A geometric examination of matured and heterogeneous HPC blade sets has been conducted. For this, over 1,400 ex-service airfoils of ten different compressor stages have been digitized by means of optical measurement and furthermore parameterized using a parametric blade model. To obtain reference geometries for all 10 stages the method has likewise been applied to 300 new production blades. In doing so, operational and manufacture-related effects on blade geometry could be differentiated. From a statistical evaluation of a variety of profile paramaters of used and new airfoils the following conclusions have been drawn:

(i) While it does not apply for all of the average characteristics of degradation throughout the HPC, relative maximum deviations of ex-service airfoils do exceed corresponding manufacture scatter in general. This is true for the entirety of the respective blade span. Whereas the standard deviations of stage 2 are similar comparing new and used blades, the stages 6 and 10 show a clear increase in standard deviation of all parameters for the used parts in relation to the averaged new part. Although this appears to reasonable taking deterioration and repair effects into account, it is contrary to the results of Goodhand et al. [3], who defined the production variations as the dominant factor.

(ii) Regarding the leading edge area, front and rear stages appear to respond differently to operation. Whereas the upstream blade LEs appear sharpened and thinned, the downstream stages feature blunted leading edges to a great extent. As a general observation, the rear blade rows were found to be more susceptible to deterioration. It can be seen that the scatter between minimum and maximum values increases with the HPC stages. The difference in profile thickness is in the front stage about $10 \%$, in the mid-stage $30 \%$ and in the aft stage $50 \%$. A similar trend is found for the leading edge thickness and the stagger angle. This behavior is also confirmed for the radial clearances, as the relative blade height of the matured airfoil sets decreases significantly with rising stage number.

(iii) Unlike expected, not all symptoms of deterioration were found to concentrate on the tip region. On the one hand, the scattering of the minimum and maximum values is increasing with blade height, on the other hand, a clear tendency of stagger angle change due to operational strains could not be verified along blade height.
Nevertheless, the scatter of stagger angle implies significant effects on blade performance and local flow field behavior for min. and max. values compared to average.

(iv) The overall condition of matured HPC airfoil geometries does not depend on deterioration due to service alone. Instead, it is more the result of a superposition of degradation effects, manufacture variance, and potential change of shape due to a variety of repairs. In addition, the composition of blade sets which have already received at least one shop visit may be arbitrary to a certain extent, since individual blades are repaired or exchanged without a documentation for the specific part. As a consequence, clear span-wise or stage-wise trends of deterioration of some of the investigated profile parameters might have remained undetected in the present investigation. Therefore, a future consideration of individual blade geometry in optimized compressor maintenance will only be feasible through a complete documentation and classification of HPC airfoils.

Acknowledgments The authors would like to thank MTU Maintenance Hannover $\mathrm{GmbH}$ for funding and for allowing the publication of this work. The authors are also very grateful to Institute of Jet Propulsion and Turbomachinery at the TU Braunschweig for a close and fruitful collaboration.

Open Access This article is distributed under the terms of the Creative Commons Attribution License which permits any use, distribution, and reproduction in any medium, provided the original author(s) and the source are credited.

\section{References}

1. Ebmeyer, C. et al.: Evaluation of total engine performance degradation based on modular efficiencies. AMSE Paper No. GT2011-45839

2. Giebmanns, A. et al.: Analyzing and optimizing geometrically degraded transonic fan blades by means of $2 \mathrm{D}$ and $3 \mathrm{D}$ simulations and cascade measurements. ASME Paper No. GT201269064

3. Goodhand, M. N., Miller Robert, J., Lung Hang, W.: The sensitivity of $2 \mathrm{D}$ compressor incidence range to in-service geometric variation. ASME Paper No. GT2012-68633

4. Holtzhausen, S. et al.: Highly accurate automated $3 \mathrm{~d}$ measuring and data conditioning for turbine and compressor blades. ASME Paper No. GT2009-59902

5. Krone, J.-H., Friedrichs, J., Städing, J.: Performance study of deterioration effects of a jet engine high pressure compressor stage. DLRK Paper No. DLRK2012-281377

6. Lange, A. et al.: Introduction of a Parameter based compressor blade model for considering measured geometry uncertainties in numerical simulation. ASME Paper No. GT2009-59937

7. Lange, A. et al.: Probabilistic CFD simulation of a high-pressure compressor stage taking manufacturing variability into account. ASME Paper No. GT2010-22484 
8. Lange, A. et al.: Impact of manufacturing variability and nonasymmetry on high-pressure compressor stage performance. ASME Paper No. GT2011-45718

9. Lange, A. et al.: Impact of manufacturing variability on multistage high-pressure compressor performance. ASME Paper No. GT2012-69571
10. Roberts, W. B. et al.: The effect of variable chord length on transonic axial rotor performance. ASME Paper No. 2001-GT0498 